\title{
Equilibrium microphase separation in the two-leaflet model of lipid membranes
}

\author{
Ramon Reigada $^{1, *}$ and Alexander S. Mikhailov ${ }^{2,3}$ \\ ${ }^{1}$ Departament de Química Física i Institut de Química Teòrica i Computacional (IQTCUB), \\ Universitat de Barcelona, Avinguda Diagonal 647, 08028 Barcelona, Spain \\ ${ }^{2}$ Abteilung Physikalische Chemie, Fritz-Haber-Institut der Max-Planck-Gesellschaft, Faradayweg 4-6, 14195 Berlin, Germany \\ ${ }^{3}$ Department of Mathematics and Life Sciences, Hiroshima University, 1-3-1 Kagamiyama, Higashi-Hiroshima, 739-8530, Japan
}

(Received 31 October 2015; revised manuscript received 20 December 2015; published 25 January 2016)

\begin{abstract}
Because of the coupling between local lipid composition and the thickness of the membrane, microphase separation in two-component lipid membranes can take place; such effects may underlie the formation of equilibrium nanoscale rafts. Using a kinetic description, this phenomenon is analytically and numerically investigated. The phase diagram is constructed through the stability analysis for linearized kinetic equations, and conditions for microphase separation are discussed. Simulations of the full kinetic model reveal the development of equilibrium membrane nanostructures with various morphologies from the initial uniform state.
\end{abstract}

DOI: 10.1103/PhysRevE.93.010401

The idea of membrane nanodomains forming lipid rafts [1], although controversial, has been useful as a basic organizing principle to understand the connection between membrane structure and functionality [2]. Despite broad experimental evidence supporting nanoscale raft organization, clear understanding of its physical origin still remains under debate [3]. Generally, there are two scenarios that may account for prevention of complete phase separation in binary solutions and development of stationary finite-size domains. At equilibrium, such domains can arise from the microphase separation resulting from the competition between attractive local and repulsive long-ranged interactions between particles [4]. In nonequilibrium systems, an analog of the microphase separation can be observed when energetically activated reactions between two components are included [5,6]. The nonequilibrium scenario has inspired a variety of proposals in the context of the cell membrane [7-12].

Equilibrium microphase separation in two-component lipid membranes was found in two-leaflet models with different lipid composition in each leaflet [13-16]. In these models, coupling between the local membrane curvature and the difference of local lipid compositions in two layers could give rise to an instability at finite wave numbers. In the resulting modulated phases, lipid variations were, however, anticorrelated between the two layers, in contrast to the correlation or domain registration, expected for lipid rafts. Recent computer simulations of molecular coarse-grained models for two-component lipid bilayers have also shown that correlated (registered) nanodomains with similar local composition in both leaflets can be observed [17]. To explain this, a mechanism that additionally allows for local changes of the membrane thickness has been proposed [17,18] and the minimization of the free energy using the Brazovskii approximation has shown that modulated phases with identical lipid composition in the leaflets are possible in this case [18].

In this Rapid Communication, the kinetic two-leaflet model for the mechanism $[17,18]$ is constructed and investigated. By direct stability analysis, general conditions for microphase separation are obtained and the phase transition diagram is determined. Nonlinear simulations of various emerging nanostructured membrane phases are further performed, demonstrating how such structures develop through the instability of the initial uniform state.

In the model, the membrane is described as consisting of two coupled laterally heterogeneous elastic surfaces (leaflets) that interact one with another. Close to the critical point, the Landau free energy (in units of $k_{B} T$ ) associated with phase segregation can be expressed in terms of composition order parameters $\left(\phi_{+}, \phi_{-}\right)$representing local differences in lipid concentrations for each leaflet,

$$
\begin{aligned}
\mathfrak{\Im}_{\text {seg }}= & \int d r^{2}\left[-\frac{\alpha}{2} \phi_{+}^{2}+\frac{\beta}{4} \phi_{+}^{4}+\frac{\gamma}{2}\left|\nabla \phi_{+}\right|^{2}\right. \\
& \left.-\frac{\alpha}{2} \phi_{-}^{2}+\frac{\beta}{4} \phi_{-}^{4}+\frac{\gamma}{2}\left|\nabla \phi_{-}\right|^{2}\right] .
\end{aligned}
$$

For positive parameters $\alpha$ and $\beta$, two phases segregate and different local curvatures develop. Instead of using the Helfrich elastic free energy of the membrane as a single sheet, we consider (see also $[17,18]$ ) the two leaflets separately, so that

$$
\begin{aligned}
\Im_{\text {elastic }}= & \frac{1}{2} \int d^{2} r\left[\sigma\left(\nabla h_{+}\right)^{2}+\kappa\left(\nabla^{2} h_{+}-c_{0,+}\right)^{2}\right. \\
& \left.+\sigma\left(\nabla h_{-}\right)^{2}+\kappa\left(\nabla^{2} h_{-}-c_{0,-}\right)^{2}\right],
\end{aligned}
$$

where $h_{+}$and $h_{-}$are height variations for the upper and lower layers (Fig. 1), $\sigma$ is the surface tension parameter, and $\kappa$ is the layer bending modulus. Spontaneous curvatures of each leaflet $\left(c_{0,+}, c_{0,-}\right)$ are determined by their local lipid composition; i.e., we have $c_{0,+}=\phi_{+} c_{0}$ and $c_{0,-}=-\phi_{-} c_{0}$. This means that since the preferred curvature of a particular lipid phase depends on the shape of the predominant lipid species, it changes its sign from one layer to the other (Fig. 1).

The two layers are coupled by an interfacial surface tension term that accounts for the energy between two different lipid domains at opposite leaflets,

$$
\mathfrak{\Im}_{\text {int }}=\frac{1}{2} \int d^{2} r\left[\xi\left(\phi_{+}-\phi_{-}\right)^{2}\right] .
$$




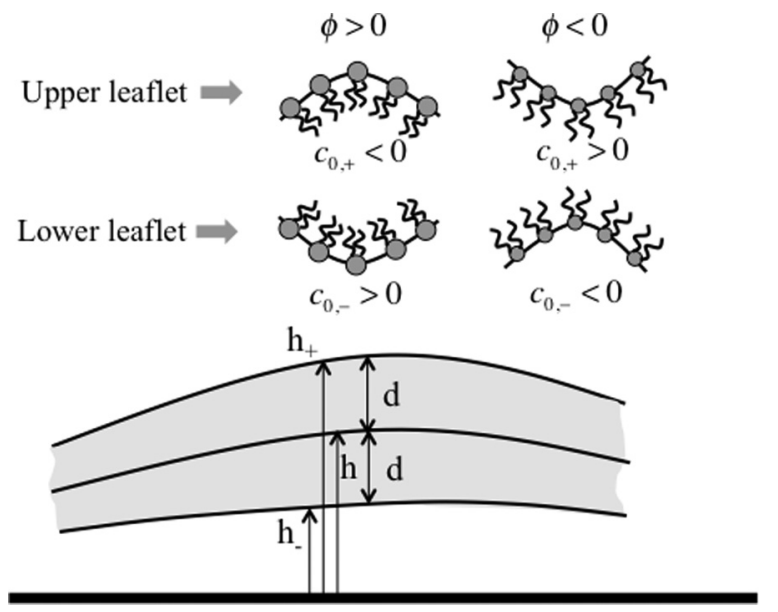

FIG. 1. (Top) Opposite spontaneous curvatures in two membrane leaflets. (Bottom) Local height and thickness variables that describe the shape of a two-leaflet membrane.

Furthermore, there is a harmonic energy penalty for the thickness fluctuations defined as half the deviation from the average membrane thickness [19,20],

$$
\Im_{\text {thick }}=\frac{1}{2} \int d^{2} r\left\{\chi\left[\left(\frac{h_{+}-h_{-}}{2}\right)-d_{0}\right]^{2}\right\},
$$

where the parameter $\chi$ characterizes the thickness stiffness of the membrane.

In the present study, only phase segregation processes that take place symmetrically in both leaflets will be considered, so that only one variable $\phi=\phi_{+}=\phi_{-}$is required and the interfacial free energy (3) vanishes.

We adopt a conserved kinetic scheme for the composition order parameter,

$$
\dot{\phi}=D \nabla^{2}\left[\frac{\delta \mathfrak{s}}{\delta \phi}\right]
$$

Evolution of the membrane shape is described by kinetic equations for the two heights,

$$
\dot{h}_{+}=-\Lambda \frac{\delta \mathfrak{s}}{\delta h_{+}} \quad \dot{h}_{-}=-\Lambda \frac{\delta \mathfrak{I}}{\delta h_{-}} .
$$

It is known that relaxation processes of the membrane shape are relatively complex because hydrodynamic solvent flows become induced [21,22]. As a result, the relaxation rate constant depends on the wave number as $\Lambda(q)=(4 \eta q)^{-1}$ where $\eta$ is the kinematic viscosity of the solvent. In the present study, the wave number dependence is, however, for simplicity neglected.

The model can be further simplified introducing the midplane height $h$ and the thickness variable $d$ instead of the two-leaflet height variables $h_{+}=h+d$ and $h_{-}=h-d$ (Fig. 1). The final kinetic equations have the form

$$
\begin{aligned}
& \dot{\phi}=D \nabla^{2}\left[\left(2 \kappa c_{0}^{2}-\alpha\right) \phi+\beta \phi^{3}-\gamma \nabla^{2} \phi-2 \kappa c_{0} \nabla^{2} d\right], \\
& \dot{d}=-\Lambda\left[2 \kappa \nabla^{4} d-2 \sigma \nabla^{2} d-\kappa c_{0} \nabla^{2} \phi+\chi\left(d-d_{0}\right)\right], \\
& \dot{h}=-2 \Lambda\left[\kappa \nabla^{4} h-\sigma \nabla^{2} h\right] .
\end{aligned}
$$

Note that the midplane height is decoupled from the other two variables and thus the membrane independently tends to become flat in the considered symmetric composition case.

The linear stability analysis of the stationary solutions $\phi(r)=\bar{\phi}$ and $d(r)=d_{0}$ with respect to small perturbations $\delta \phi, \delta d \propto \exp [\omega(q) t+i q r]$ can be obtained straightforwardly from the linearized equations and the corresponding linearization matrix

$$
L=\left(\begin{array}{cc}
q^{2}\left[\alpha-2 \kappa c_{0}^{2}-\gamma q^{2}\right] & -2 \kappa c_{0} q^{4} \\
-2 \Lambda \kappa c_{0} q^{2} & -\Lambda\left[\chi+2 \kappa q^{4}+2 \sigma q^{2}\right]
\end{array}\right)
$$

For a tensionless membrane with $\sigma=0$, a Turing bifurcation is obtained. The first unstable mode has the wave number

$$
q_{0}^{2}=\frac{2 \alpha-3 \kappa c_{0}^{2} \pm c_{0} \sqrt{\kappa\left(9 \kappa c_{0}^{2}-4 \alpha\right)}}{2 \gamma},
$$

and the instability is found at the bifurcation boundary determined by

$$
\chi_{0}^{ \pm}=\frac{\kappa}{\gamma^{2}}\left[9 \kappa c_{0}^{2}\left(2 \alpha-3 \kappa c_{0}^{2}\right)-2 \alpha^{2} \pm 8 \sqrt{\kappa c_{0}^{2}\left(\frac{9}{4} \kappa c_{0}^{2}-\alpha\right)^{3}}\right]
$$

The phase diagram in the $(\chi, \alpha)$ parameter space is displayed in Fig. 2. Characteristic dependences of the increments of growth on the wave number $q$ in different parts of the phase diagram are shown in Fig. 3. The cusp separating regions II and III from regions I and IV is formed by the curves $\chi=\chi_{0}^{+}(\alpha)$ and $\chi=\chi_{0}^{-}(\alpha)$ determined by Eq. (10). The vertical line, separating regions I and IV and regions II

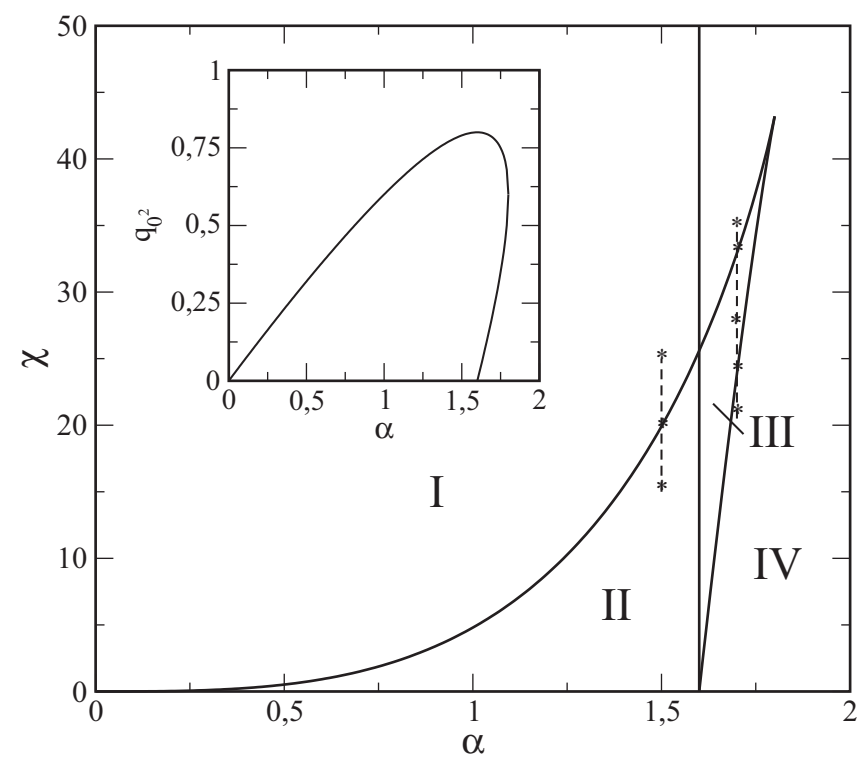

FIG. 2. The phase diagram in the plane $(\chi, \alpha)$ for $\kappa=20, c_{0}=$ 0.2 , and $\gamma=1$. Region I: no phase separation; region II: microphase separation; region III: coexistence of micro- and macrophase separation; region IV: macroscopic phase separation (spinodal decomposition). (Inset) The wave number of the critical mode of the Turing bifurcation as a function of parameter $\alpha$. 


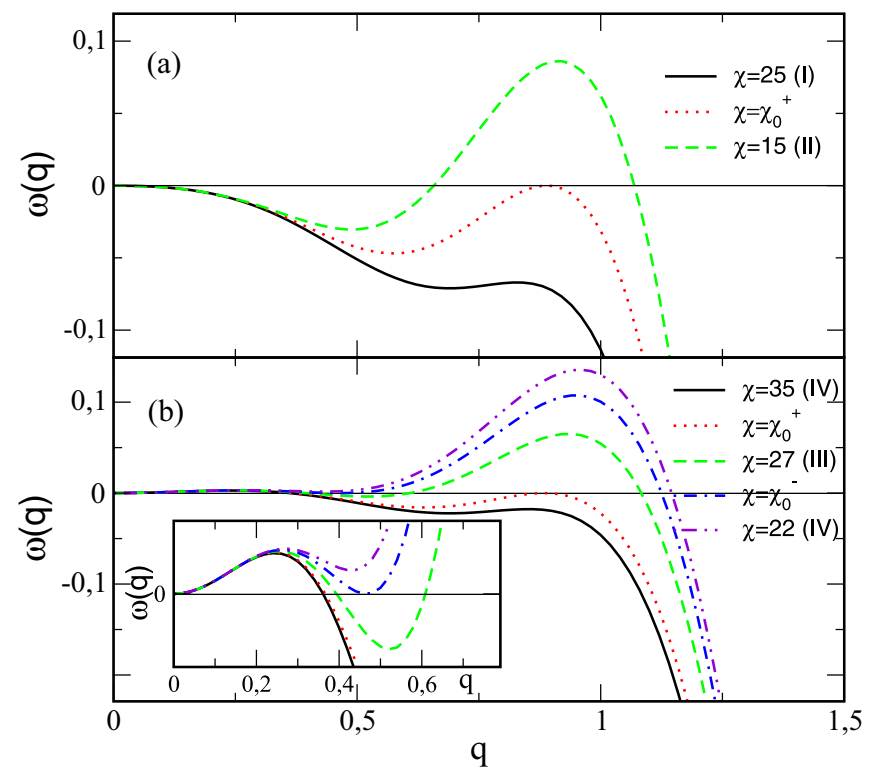

FIG. 3. Increments of growth $\omega$ as functions of the wave number $q$ at different values of parameter $\chi$ for (a) $\alpha=1.5$ and (b) $\alpha=1.7$. The choices of $\chi$ correspond to the vertical dashed lines in Fig. 2. (Inset) Enlargement of panel (b) for small wave numbers. Other parameters are $\kappa=20, c_{0}=0.2$, and $\gamma=1$.

and III, lies at $\alpha=\alpha_{\text {seg }}=2 \kappa c_{0}^{2}$. Macroscopic phase separation (i.e., spinodal decomposition) takes place in region IV, whereas microphase separation is predicted in region II. The uniform state is stable and phase separation is absent in region I. The linear stability analysis predicts for region III that the spinodal decomposition and microphase separation should coexist [see Fig. 3(b)]. Nonlinear numerical simulations in this region reveal (see below), however, that the spinodal decomposition phenomenon similar to that in region IV finally sets in this region after initial transients.

If thickness stiffness $\chi$ of the lipid bilayer is high, macroscopic phase separation is found above $\alpha=\alpha_{\text {seg }}$, whereas the uniform state is stable below this boundary. This limit corresponds to the classical Helfrich description: the thickness of the membrane cannot significantly change and the shapes of the two leaflets are almost indistinguishable. When the parameter $\chi$ is decreased, the two leaflets are, however, less strongly bound one to another and local membrane thickness may substantially vary. According to our results, classical spinodal decomposition should then be preceded by microphase separation where equilibrium domains of fixed sizes are formed (region II).

To determine final equilibrium patterns in different regions of the phase diagram, numerical simulations of Eq. (7) have been performed. A grid with $100 \times 100$ points with periodic boundary conditions was used. The mesh size was $\Delta x=$ 1, spatial derivatives were calculated employing a simple centered scheme, and a first order Euler algorithm with time step $\Delta t=0.0005$ was used for temporal integration. Simulations were started from a uniform state with $\phi(r)=$ $\bar{\phi}=0, d(r)=d_{0}$ that was randomly perturbed by applying small local variations. (a)
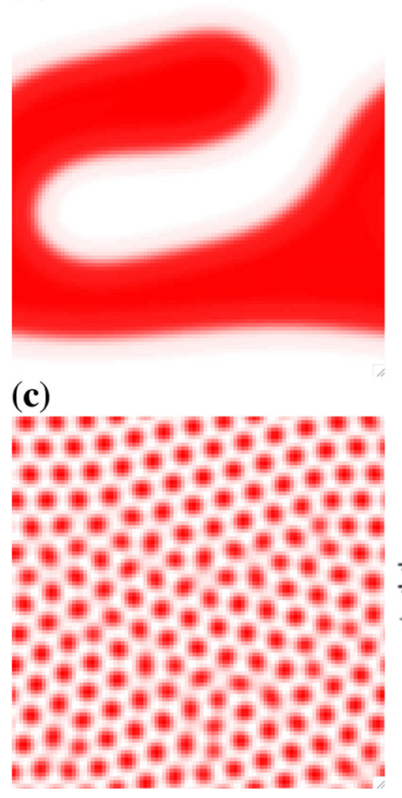

(b)

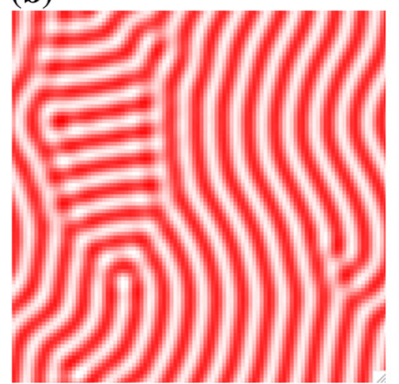

(d)

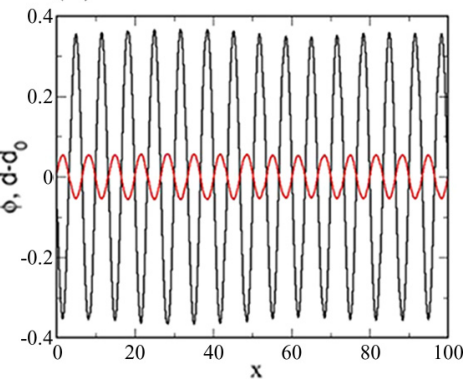

FIG. 4. (a-c) Examples of equilibrium patterns for membrane composition $\phi$ : (a) complete phase segregation (region IV, $\alpha=$ 1.75, $\chi=35, \bar{\phi}=0$ ); (b) lamellae (region II, $\alpha=1.5, \chi=15, \bar{\phi}=$ $0)$; (c) spots $\alpha=1.5, \chi=15, \bar{\phi}=0.15$ ). Panel (d) shows profiles of composition order parameter (thick) and thickness variation (thin) in a one-dimensional simulation for $\alpha=1.5, \chi=15, \bar{\phi}=0$. Other parameters are the same as in Fig. 1. The linear size of the system is 100 dimensionless length units corresponding to $200 \mathrm{~nm}$.

The simulation results are summarized in Fig. 4. A uniform stable phase and macroscopic phase separation could be confirmed for regions I and IV. In region II, microphase segregation was getting rapidly achieved. In region III, a pattern of finite-wavelength domains was developing first. Later, however, it was replaced by large homogeneous domains and complete spinodal decomposition was finally found within this region (Fig. 5).

Figures 4(b) and 4(c) show two typical morphologies (lamellae and spots) of asymptotic patterns observed under microphase separation conditions in numerical simulations for region II. Within each pattern, both local composition and membrane thickness are modulated, as illustrated in Fig. 4(d) where profiles of these variables in the one-dimensional simulation with the same parameters as in Fig. 4(b) are displayed. We checked that the characteristic length of these equilibrium patterns was close to the wavelength $\sim \pi q_{0}^{-1}$ of the critical Turing mode in Eq. (9). Patterns with characteristic length scales in the interval from 5 to 20 were found for $0.15<\alpha<\alpha_{\text {seg }}$.

In our simulations, model parameters were chosen to agree with the actual values for biological membranes. For membranes composed of phospholipids, bending rigidities $\kappa$ of a few tens of $k_{B} T$ are typical [23] and for cell membranes containing a large molar fraction of cholesterol even higher bending rigidities are possible [24]. Hence, values of $\kappa$ between 10 and 30 are realistic. The parameter $\chi$ could previously be estimated from molecular dynamics simulations as $\chi=4 \times 10^{-21} \mathrm{~J} / \mathrm{nm}^{4}$, or $\chi \approx 1 k_{B} T / \mathrm{nm}^{4}$ [19]. 


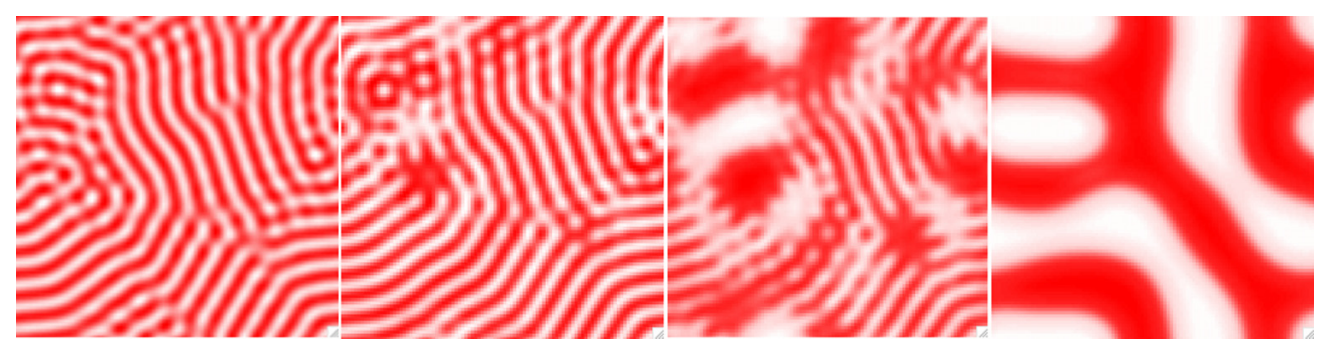

FIG. 5. Four subsequent snapshots (from left to right, ending at $t=5 \times 10^{4} t_{u}$ ) showing evolution of the local membrane composition inside region III $(\alpha=1.7, \chi=27, \bar{\phi}=0)$. Other parameters and the size of the system are the same as in Fig. 4.

The membrane thickness stiffness can be also estimated as $\chi=K_{A} / 2 d_{0}^{2}$ [20], where $K_{A}$ is the membrane compressibility modulus. A typical experimental value of $K_{A} \approx 200 \mathrm{mN} / \mathrm{m}$ [25] and an average membrane thickness $d_{0} \approx 3 \mathrm{~nm}$ lead to $\chi \approx 2 k_{B} T / \mathrm{nm}^{4}$. According to the Cahn-Hilliard theory [26], $\gamma \approx u l_{0}^{2} / 2$ where the interaction energy is about $u=1 k_{B} T$ and the characteristic interface width is $l_{0}=2-3 \mathrm{~nm}$. By choosing $\gamma=1$, the length unit in our equations becomes fixed to about $l_{u}=2 \mathrm{~nm}$, thus implying $\chi \approx 15-30 k_{B} T / l_{u}^{4}$. For the spontaneous curvature radius, estimates between 2 and $20 \mathrm{~nm}$ (namely, between 1 and $10 l_{u}$ ) are available [27], so that $c_{0}=0.1-1 l_{u}^{-1}$. Requiring that the diffusion constant is $D=1 l_{u}^{2} / t_{u}=1 \mu \mathrm{m}^{2} / \mathrm{s}$, the time unit in our model is fixed to $t_{u}=4 \times 10^{-6} \mathrm{~s}$. Under these numerical values, the microphase separation region II in the phase diagram (Fig. 2) is readily accessible and equilibrium microphase separation with characteristic length scales about a few tens of nanometers can be found.

The basic mechanism of microphase separation can also be revealed by a simple analysis (cf. [18]). Applying Eqs. (1), (2), and (4) to modulated periodic phases with wave numbers $q$ and amplitudes $\phi_{m}, d_{\mathrm{m}}$ for the composition and thickness variables, respectively, the following expression for the free energy is obtained:

$$
\begin{aligned}
\Im(q)= & -\frac{\alpha}{4} \phi_{\mathrm{m}}^{2}+\frac{3 \beta}{16} \phi_{\mathrm{m}}^{4}+\frac{\gamma}{4} \phi_{\mathrm{m}}^{2} q^{2} \\
& +\frac{\chi}{4} d_{\mathrm{m}}^{2}+\kappa\left(d_{\mathrm{m}} q^{2}-c_{0} \phi_{\mathrm{m}}\right)^{2} .
\end{aligned}
$$

In the limit of large $\chi$, variations of membrane thickness are suppressed and the minimum of free energy (11) is achieved at large wavelengths. On the other hand, for small $\chi$, membrane thickness can be modulated and the equilibrium is reached when the energy associated with the curvature [the last term in Eq. (11)] is minimized, leading to $d_{m}=c_{0} \phi_{m} / q^{2}$. Then, the free energy (11) reads

$$
\Im(q)=-\frac{\alpha}{4} \phi_{m}^{2}+\frac{3 \beta}{16} \phi_{m}^{4}+\frac{\gamma}{4} \phi_{m}^{2} q^{2}+\frac{\chi c_{0}^{2}}{4 q^{4}} \phi_{m}^{2},
$$

and its minimum is achieved for the modulated phase with the finite wave number $q=\left(2 \chi c_{0}^{2} / \gamma\right)^{1 / 6}$. Note that this agrees with Eqs. (9) and (10) that yield the same critical wave number on the instability boundary when $\alpha$ (and therefore $\chi$ ) are small.
Thus, either macro- or microphase separation takes place depending on the competition between domain coarsening and thickness modulation. At short wavelengths, the preferred curvature can be locally achieved without implying large thickness energy penalties.

In summary, we have constructed and investigated a kinetic model for equilibrium microphase separation in twocomponent lipid membranes that corresponds to the mechanism based on the coupling between the local lipid composition and thickness variation of the membrane $[17,18]$. The instabilities of the uniform state leading to the formation of the spatially modulated phases have been identified and the phase diagram of the model has been determined. Direct simulations of the kinetic model have been performed revealing the development of various equilibrium nanostructures. In the region III where the uniform state is unstable both for spinodal decomposition and for the development of modulated structures, numerical simulations have revealed that first the modulated phase develops but then it is replaced by macrophase separation with large domains.

In our study, fluctuations in the membrane geometry and local lipid composition have not been taken into account, although they may be substantial for soft structures on nanoscales. They can be further taken into account by introducing appropriate noise terms in the kinetic equations (cf. [28]). Previous investigations of the effects of thermal fluctuations on nonequilibrium Turing-like nanostructures and traveling waves have shown that, instead of periodic structures, irregular lamellae arrays and isolated fragments are observed [29,30]. Similar behavior can be expected for the considered lipid membranes. Due to thermal fluctuations, small domains may also repeatedly break and rearrange due to thermal fluctuations and thus have finite lifetimes, instead of representing infinitely stable equilibrium structures. Combined with the effects of transmembrane proteins favoring a specific hydrophobic thickness, such phenomena may lead to a rich dynamic behavior of biological membranes and this can be an interesting topic for further studies.

This work has been supported by the spanish Ministerio de Economía y Competitividad through Project No. BFU201021847-C02-02. We are grateful to S. Komura for useful discussions.
[1] K. Simons and E. Ikonen, Science 290, 1721 (2000).

[2] A. D. Douglass and R. D. Vale, Cell 121, 937 (2005).
[3] J. F. Hancock, Nat. Rev. Mol. Cell Biol. 7, 456 (2006).

[4] M. Seul and D. Andelman, Science 267, 476 (1995). 
[5] S. C. Glotzer, E. A. Di Marzio, and M. Muthukumar, Phys. Rev. Lett. 74, 2034 (1995).

[6] A. S. Mikhailov and G. Ertl, ChemPhysChem 10, 86 (2009).

[7] M. S. Turner, P. Sens, and N. D. Socci, Phys. Rev. Lett. 95, 168301 (2005).

[8] J. Gómez, F. Sagués, and R. Reigada, Phys. Rev. E 80, 011920 (2009).

[9] R. Reigada, J. Buceta, and K. Lindenberg, Phys. Rev. E 71, 051906 (2005).

[10] B. B. Machta, S. Papanikolaou, J. P. Sethna, and S. L. Veatch, Biophys. J. 100, 1668 (2011).

[11] J. Gómez, J. Buceta, and R. Reigada, Sci. Rep. 3, 2608 (2013).

[12] C. Zurzolo, G. van Meer, and S. Mayor, EMBO Rep. 4, 1117 (2003).

[13] P. L. Hansen, L. Miao, and J. H. Ipsen. Phys. Rev. E 58, 2311 (1998).

[14] M. Schick, Phys. Rev. E 85, 031902 (2012).

[15] G. Guegen, N. Destainville, and M. Manghi. Eur. Phys. J. E 37, 76 (2014).

[16] Y. Hirose, S. Komura and D. Andelman, Phys. Rev. E 86, 021916 (2012).
[17] S. Meinhardt, R. L. C. Vink, and F. Schmid. Proc. Natl. Acad. Sci. USA 110, 4476 (2013).

[18] F. Schmid, S. Dolezel, O. Lenz, and S. Meinhardt, J. Phys: Conf. Ser. 487, 012004 (2014).

[19] E. Lindahl and O. Edholm, Biophys. J. 79, 426 (2000).

[20] G. Brannigan and F. L. H. Brown, Biophys. J. 90, 1501 (2006).

[21] H-Y. Chen and A. S. Mikhailov, Phys. Rev. E 81, 031901 (2010).

[22] U. Seifert, Adv. Phys. 46, 13 (1997).

[23] M. Kummrow and W. Helfrich, Phys. Rev. A 44, 8356 (1991).

[24] F. Brochard and J. F. Lennon, J. Phys. (Paris) 36, 1035 (1975).

[25] D. Marsh, Chem. Phys. Lipids 144, 146 (2006).

[26] J. W. Cahn and J. E. Hilliard, J. Chem. Phys. 28, 258 (1958).

[27] H. Wang, D. Hu, and P. Zhang, Commun. Comput. Phys. 13, 1093 (2013).

[28] M. Hildebrand and A. S. Mikhailov, J. Phys. Chem. 100, 19089 (1996)

[29] M. Hildebrand, A. S. Mikhailov, and G. Ertl, Phys. Rev. Lett. 81, 2602 (1998).

[30] M. Hildebrand, A. S. Mikhailov, and G. Ertl, Phys. Rev. E 58, 5483 (1998). 